\title{
Muon science in J-PARC
}

\author{
N. Kawamura ${ }^{a}$, P. Strasser ${ }^{a}$, S. Makimura ${ }^{a}$, \\ K. Nishiyama ${ }^{a}$, R. Kadono ${ }^{a}$, K. Shimomura ${ }^{a}$, \\ K. Nagamine ${ }^{a, 1}$, W. Higemoto ${ }^{b}$, J.L. Beveridge ${ }^{a}$, K. Ishida ${ }^{c}$ \\ Y. Miyake ${ }^{a}$, \\ and Materials and Life Science Facility group ${ }^{a, b}$ \\ ${ }^{a}$ High Energy Accelerator Research Organization, \\ Tsukuba, Ibaraki 305-0801, Japan \\ ${ }^{b}$ Japan Atomic Energy Research Institute, \\ Tokai, Ibaraki 319-1195, Japan \\ ${ }^{c}$ RIKEN, Wako, Saitama 351-0198, Japan
}

\begin{abstract}
The world strongest pulsed muon facility will be established in 2007 as one of five experimental facilities in J-PARC (Japan Proton Accelerator Research Complex). From the muon target irradiated by 1-MW proton beam $(3 \mathrm{GeV} \times 333 \mu \mathrm{A}, 25 \mathrm{~Hz}$ repetition), intense muon beam can be provided to four secondary muon beam lines. Each secondary line is designed to extract characteristic muon beam to be utilized for various kinds of muon science.
\end{abstract}

\section{Outline}

The project of a new high intensity proton accelerator has been jointly progressed between JAERI and KEK. This project, called J-PARC (Japan Proton Accelerator Research Complex), is in a stage of construction since 2001 toward the first beam in 2007.

An accelerator complex of J-PARC consists of a 400-MeV linac, 3-GeV rapid cycling synchrotron (RCS) and 50-GeV main synchrotron ring (MR). About $90 \%$ of $3-\mathrm{GeV}, 333-$ $\mu \mathrm{A}(1 \mathrm{MW})$ beam is transported to Material and Life Science Facility (MLF), and the remain of proton beam is sent to MR to accelerate and deliver to the hadron facility and the neutrino beam line. Accelerator-driven transmutation experimental facility is also planed to be constructed at the extension of the $400-\mathrm{MeV}$ linac.

\footnotetext{
${ }^{1}$ Present Address: RIKEN, Wako, Saitama 351-0198, Japan
} 


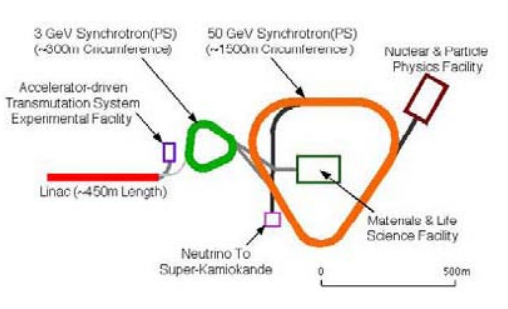

High Intensity Proton Accelerator Project

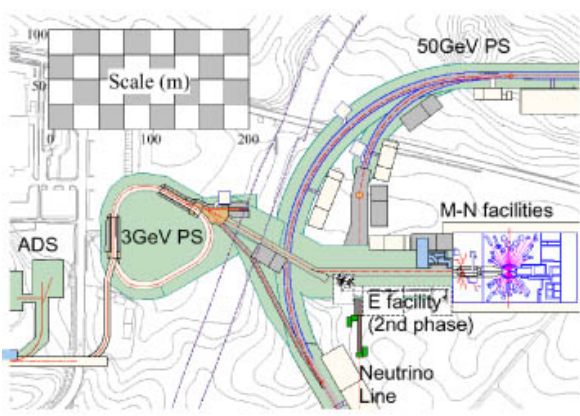

Figure 1: Accelerators in J-PARC

MLF is located at the central place of MR, and consists of Muon Science Facility and Japan Spallation Neutron Source (JSNS). The muon target is located about 30-m upstream of the neutron target. This tandem design is similar to ISIS proton synchrotron facility, Rutherford Appleton Laboratory, UK, in compare with present Booster synchrotron facility, KEK, where the muon and neutron facility have their own proton beam dump and switch the beam time periodically. In J-PARC, 3-GeV proton beam passes through the muon target and then reaches the neutron target, and thus both secondary muon beam and neutron beam are obtained simultaneously.

\section{Beam line design}

As shown in Fig. 2, the proton is transported from the left side to the right side of the neutron target through the muon target. The secondary beam lines of Muon Science Facility are extracted from the target; two lines are at 60 degree (forward) with respect to the primary beam, and the other two are at 135 degree (backward).

The forward lines are designed to transport 'decay muon'. The front magnets transport pions to decay section of solenoid magnet, and then either positive or negative muons are transported to experimental areas. Two backward lines are designed to transport 'surface muons', which originate from pions stop at around the target surface. Surface muons have monochromatic momentum of $30 \mathrm{MeV} / \mathrm{c}$. Because the primary beam has a time structure with two bunches, muon beam has a double pulse structure in a beam spill; 100-ns full-width pulse 600-ns apart from each other. In order to make a single pulse or to make a shorter pulse, we will adopt a kicker or slicer system.

One of the decay-muon lines (upper right) will be utilized to obtain relatively high momentum muon beam up to more than a few hundreds $\mathrm{MeV} / \mathrm{c}$, while the conventionaldesign decay-muon line (lower right) transports up to around $100 \mathrm{MeV} / \mathrm{c}$. A plan exists for the lower left beam line to construct a large solid angle axial focusing superconducting surface muon channel, which was developed at KEK [2]. However, in the first phase of J-PARC construction, the muon group decided to concentrate on the construction of the 
conventional decay muon beam line, and then a surface muon beam line.

Due to severe effect of radiation around the muon target and its downstream, the primary beam line is constructed in a 14-m-width tunnel. The tunnel is filled with the beam line components, such as the target chamber, the magnets and radiation shields. After beam is on, hand-on maintenance of those devices becomes very difficult. Thus, the design of beam line have to be fixed for some extent even for beam lines constructed in the second phase or later. Namely, the beam optics of a beam line constructed in future is restricted. On the other hand, because the effect of residual activity is not serious in the upstream of the muon target, we prepared a target station space and two secondary beam line tunnels at about 15 -m upstream.

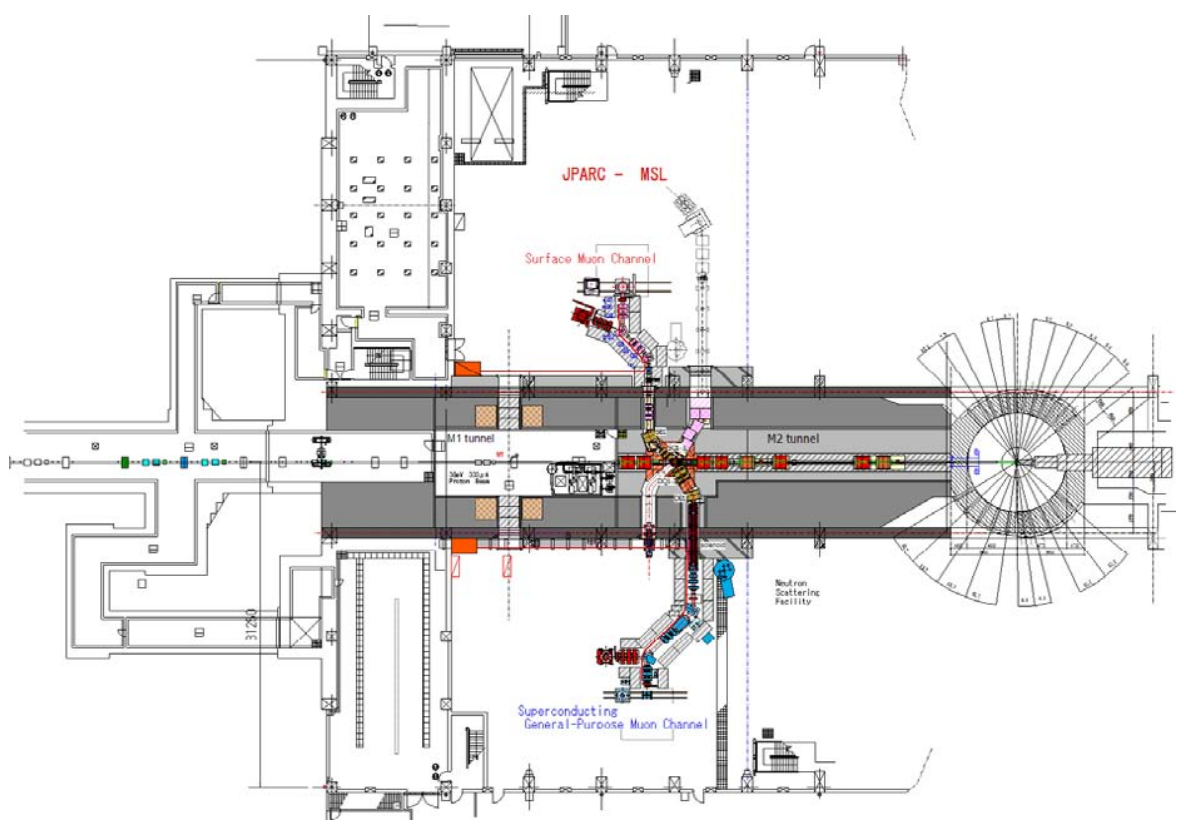

Figure 2: Plan view of Material and Life Science Facility

\section{Muon yield}

We adopt 2-cm thickness graphite, of which density is $1.8 \mathrm{~g} / \mathrm{cm}^{3}$, as the pion production target. In this case, more than $90 \%$ of proton beam is guaranteed to be transported to the neutron target. About $2 \%$ of $3-\mathrm{GeV}$ proton yields pion. Taking into account the solid angle of injector magnet and transmission efficiency of the beam line, the muon yield at the experimental area is evaluated to be $5 \times 10^{6} \mu^{+} / \mathrm{s}$ and $1 \times 10^{6} \mu^{-} / \mathrm{s}$ for around 60 $\mathrm{MeV} / \mathrm{c}$ with $10 \%$ momentum spread at the decay-muon beam line, $1 \times 10^{7} \mu^{+} / \mathrm{s}$ at the 
surface-muon beam line. Comparable number of surface muons are also available in the decay-muon beam line. The beam spot size is about $25 \mathrm{~mm}$, almost the same as that of primary beam on the pion-production target. These muon intensity corresponds to about ten times lager than those of the RIKEN-RAL Muon Facility [3].

\section{Muon science in J-PARC}

Scientific programs towards J-PARC has been discussed in the J-PARC Muon Science Advisory Committee (MuSAC), which is organized under the J-PARC Project Director during construction period [4]. The committee has been organized since 2003, and reviewed and advised both project definition of the facility and content of the 1st experimental program.

The committee discussed the following programs in addition to condensed matter studies using muon spin as a probe:

- muon catalyzed fusion $(\mu \mathrm{CF})$ study in high temperature and high pressure solid

- resonant formation study in $\mu \mathrm{CF}$ under controlling initial $\mathrm{D}_{2}$ state.

- studies of $\mu \mathrm{CF}$ processes by X-ray measurements

- negative muon lifetime and a muon capture rate in liquid hydrogen

- $\mathrm{Mu}-\overline{\mathrm{Mu}}$ conversion

- high precision spectroscopy of muonium atomic levels

User programs can be started from 2009 after beam commissioning in 2008. Before that, more serious discussion about the 'day-one' experiments have to be done, taking into account design of beam line and also experimental area.

The overall information about J-PARC can be obtained in the reference [5].

\section{References}

[1] Y. Miyake et al., Hyp. Int. 138 (2001) 475.

[2] H. Miyadera et al., Physica B 326 (2003) 265.

[3] T. Matsuzaki et al., Nucl. Inst. and Meth. A 465 (2001) 365.

[4] KEK Proceedings 2002-28.

[5] The Joint Project for High-Intensity Proton Accelerators, KEK Report 99-4; JAERITech 99-056; JHF-99-3 (1999). The present status of J-PARC is available in the official web site, http://www.j-parc.jp/. 